Mathilde Hennig (Gießen)

\title{
Grammatik und Variation im Spannungsfeld von Sprachwissenschaft und öffentlicher Sprachreflexion
}

That language is variable is a matter of everyday observation. (Durrell 2004, S. 195)

\begin{abstract}
Der Beitrag bezieht systematische und funktionale Faktoren zur Erklärung grammatischer Variation aufeinander, indem er ausgehend von der Annahme eines rekursiven Systems mit konfligierenden Teilsystemen ,System als Möglichkeitsraum für (funktional ausdifferenzierte) Variation versteht. Inwiefern die vom System bereitgestellten Möglichkeiten grammatischer Variation im Sprachgebrauch genutzt werden, diskutiert der Beitrag anhand der lexikographischen Praxis der Erfassung von grammatischer Variation im Dudenband 9 „Richtiges und gutes Deutsch“. Mit diesem Material werden nicht nur zentrale Bereiche grammatischer Variation rekonstruiert, sondern auch Zentralbereiche grammatischer Variation mit diasystematischen Variationsdimensionen korreliert.
\end{abstract}

\section{Einleitung}

Grammatische Variation ist sowohl Gegenstand linguistischer Betrachtung als auch öffentlicher Sprachreflexion. Gerade im Bereich der grammatischen Variation zeigt sich besonders deutlich, dass Linguisten und Nichtlinguisten sich den Gegenstand ,Sprache“ teilen. Die linguistische Beschäftigung mit grammatischer Variation wird nicht nur begünstigt durch das Erstarken variationslinguistischer Forschungsrichtungen in den letzten Jahrzehnten und die inzwischen stark ausgebauten korpuslinguistischen Möglichkeiten, sondern fügt sich auch gut ein in die Bemühungen der germanistischen Linguistik, ihrer Bringschuld gegenüber der Gesellschaft gerecht zu werden. Der vorliegende Beitrag setzt an der Überlegung an, dass es in diesem Kontext sinnvoll sein könnte, nicht nur der Frage nachzugehen, was wir als Linguisten der Öffentlichkeit diesbezüglich bieten können, sondern auch umgekehrt die öffentliche Sprachreflexion als eine Fundgrube für unser Verständnis von grammatischer Variation zu verstehen. 
Die Wahrnehmung von grammatischen Varianten bildet sowohl den Ausgangspunkt linguistischer als auch nicht-linguistischer Auseinandersetzung mit grammatischer Variation. In der nicht-linguistischen Beschäftigung mit Sprache ist sie geprägt von den jeweiligen Benutzungskontexten im natürlichen, alltäglichen Sprachgebrauch. Die linguistische Beschäftigung mit grammatischer Variation hingegen ist in verschiedenste linguistische Teildisziplinen eingebettet. Das große Repertoire an Forschungsrichtungen - Regionalsprachenforschung, Areallinguistik, Soziolinguistik, Gesprochene-Sprache-Forschung, Interaktionale Linguistik, Internetkommunikationsforschung, Medientheorie etc. sowie Grammatikforschung, Zweifelsfälleforschung, Korpuslinguistik - kann als Indiz dafür verstanden werden, dass grammatische Variation als ein Zentralthema der germanistischen Linguistik des 21. Jahrhunderts aufgefasst werden kann. Vor dem Hintergrund der zentralen Bedeutung von grammatischer Variation als eines der Kernthemen der aktuellen Linguistik sowie als Bindeglied zwischen Wissenschaft und Gesellschaft möchte der vorliegende Beitrag dazu anregen, trotz guter Gründe für die Spezialisierung unserer Wissenschaftsdisziplin auch übergreifenden Fragen nach dem grundsätzlichen Stellenwert grammatischer Variation und allgemeinen Gründen für grammatische Variation nachzugehen. Dabei hilft möglicherweise - so die These - die Rückbesinnung auf den nicht durch den disziplinären Kontext perspektivgeleiteten, sondern rein handlungsfunktional bestimmten Umgang mit grammatischer Variation im alltäglichen Sprachgebrauch.

Dem Umgang mit grammatischer Variation im alltäglichen Sprachgebrauch nähert sich der vorliegende Beitrag über die Sprachberatung und greift dabei insbesondere auf die Sprachberatungspraxis der Dudenredaktion zurück sowie auf die daraus hervorgegangene lexikographische Praxis der Erfassung von (grammatischen) Variationsphänomenen im Dudenband 9 „Richtiges und gutes Deutsch“. Die Sprachberatung bietet uns Linguisten einen Zugang zur Wahrnehmung grammatischer Variation in der öffentlichen Sprachreflexion. Da es bislang aber keine systematische Archivierung von Sprachberatungsfragen gibt, bietet die auf der Sprachberatung der Dudenredaktion aufbauende lexikographische Erfassung der Fragen im Dudenband 9 einen hilfreichen Zugang zu dieser Form von Daten, die zwar keine Sprachgebrauchsdaten im engeren Sinne sind, aber doch einen unmittelbaren Bezug zu Sprachgebrauchskontexten haben. Aufgrund der Breite des Materials im Dudenband 9 wird in Kapitel 3 der Versuch unternommen, ein Bild von der Wahrnehmung grammatischer Variation in der öffentlichen Sprachreflexion zu zeichnen. Kapitel 2 bietet den theoretischen Hintergrund für die Ausarbeitung der Fragestellungen, die die Grundlage der Beschäftigung mit dem genannten Datenmaterial bieten sollen. Dabei geht es einerseits darum, funktionale und systematische Ansätze zur Erklärung grammatischer Variation gegen- 
überzustellen, vor allem aber auch darum, mit dem auf Ágel (2008) zurückgehenden Begriff der konfligierenden Teilsysteme eine Erklärung für systematische Zusammenhänge zwischen System und Variation zu erarbeiten.

\section{Zur Rolle des Systems als Möglichkeitsraum für grammatische Variation}

Die Beschäftigung mit grammatischer Variation in der Linguistik umfasst ein breites Spektrum: Es reicht von spezifisch gesprochensprachlichen Varianten wie dem zu Berühmtheit gelangten weil+Verbzweit über dialektale Varianten wie flektierte Subjunktoren bis hin zu weniger diasystematisch verankerten Varianten wie die Varianten der Pluralbildung oder der Kennzeichnung des Genitivs. Es lassen sich zwei zentrale Linien der Erklärung grammatischer Varianten beobachten:

1. Die sprachsystematische Erklärung grammatischer Varianten auf der Basis von systemimmanenten Faktoren: Ein Beispiel ist der Einfluss der lautlichen und morphologischen Struktur von Substantiven auf die Kennzeichnung des Genitivs (die wichtigsten Varianten sind hier -s und -es), vgl. dazu Konopka/ Fuß (2016).

2. Die funktionale Erklärung grammatischer Varianten auf der Basis von außerlinguistischen Faktoren wie Gruppenzugehörigkeit, interaktionale Faktoren, bestimmte Anforderungen bestimmter Kommunikationsbereiche etc.

Gerade die funktionale Erklärung hat aufgrund der zunehmenden Ausdifferenzierung variationslinguistischer Forschungsrichtungen sowie des Erstarkens variationslinguistischer Forschung allgemein derzeit Hochkonjunktur. Dabei findet die funktionale Erklärung grammatischer Variation stets im Kontext einzelner variationslinguistischer Forschungsrichtungen statt. Damit wird von vornherein ein Zusammenhang zwischen grammatischer Variation und der Diasystematik sprachlicher Variation hergestellt. Die Erklärung grammatischer Varianten durch im weitesten Sinne pragmatische Faktoren passt sicherlich nicht zuletzt deshalb ins aktuelle linguistische Zeitbild, weil damit dem Handlungscharakter von Sprache begegnet werden kann. Ich möchte im vorliegenden Beitrag aber gerade vor dem Hintergrund der dominanten Rolle funktionaler Erklärungen die Aufmerksamkeit auf den Anteil des Systems an grammatischer Variation lenken. So gehe ich davon aus, dass das System nicht einfach nur als ein der funktionalen Ebene untergeordnetes ausführendes Organ verstanden werden sollte, sondern dass das System eine entscheidende Rolle in Bezug auf grammatische Variation spielt, indem es diese überhaupt erst ermöglicht. 
Wie auch in Hennig (i.Vorb.) greife ich zur Beschäftigung mit der Frage nach grundsätzlichen Zusammenhängen zwischen System und Variation auf den Systembegriff Coserius zurück (1971, S. 67). Coserius Systembegriff ist ein sprachtheoretischer Begriff, der hier insofern von Belang ist, als das grammatische System ein Teil des Systems einer Einzelsprache ist. Der Systembegriff Coserius bietet m.E. deshalb eine geeignete Grundlage für die Frage nach der Rolle des Systems an einzelsprachlicher Variation, weil ,System bei Coseriu über die intermediäre Ebene der Norm systematisch an das Sprechen rückgekoppelt ist. Es handelt sich hier also nicht um ein ,System vs. Gebrauch'-Gegensatzpaar, sondern das System ist als Abstraktion aus dem Sprechen nicht ohne dieses denkbar. ${ }^{1}$ Andererseits sind Sprechen und Norm auch nicht unabhängig vom System (quasi als dieser Abstraktionsstufe vorgeschaltete Ebenen), sondern das System bietet vielmehr den Möglichkeitsraum, in dem Varianten überhaupt normal sein und somit im Sprechen angewendet werden können. ${ }^{2}$

Für die hier zu führende Diskussion ist darüber hinaus der von Coseriu herausgearbeitete Zusammenhang zwischen der Struktur und der Architektur der Sprache zentral:

Nur für die homogene funktionelle Sprache stellt sich die Frage nach der Strukturiertheit im engeren Sinne, d.h. die Frage nach den stabilen Formen der inneren Relationen. Hier können wir fragen, wie die Technik einer funktionellen Sprache strukturiert ist, und die Ebenen ihrer Strukturierung feststellen, die wir Sprachnorm, Sprachsystem und Sprachtypus nennen. (Coseriu 2007, S. 265)

Hier bezieht Coseriu den Begriff des Sprachsystems auf Varietäten (bei ihm: „funktionelle Sprachen“). Sprachsysteme sind bei ihm z.B. Mundarten, Sprachniveaus und Sprachstile. Indem Coseriu den Systembegriff auf die Ebene der funktionellen Sprachen bezieht, stellt er bereits grundsätzlich einen Zusammenhang zwischen System und Variation her: „Eine historische Sprache ist nie ein einziges Sprachsystem, sondern ein Gefüge von - teilweise - verschiedenen Sprachsystemen“ (ebd., S. 24). In diesem Zitat bezieht Coseriu den Begriff des Sprachsystems aber nicht nur auf die Ebene der funktionellen Sprachen, sondern auch auf die historische Sprache (= Einzelsprache). Schmidt/Herrgen definieren Varietä-

1 In Coserius „Theorie des Sprechens“ ist - wie die Bezeichnung bereits andeutet - das Sprechen primär. Der Systembegriff ist deshalb an das Sprechen rückgekoppelt.

2 Natürlich gibt es auch Phänomene, die normal sind, obwohl sie eigentlich systemwidrig sind (vgl. die Beispiele in Ágel 2008, S. 67: eines Nachts, meines Erachtens nach). Der Großteil normaler Varianten dürfte sich aber innerhalb der Systemgrenzen bewegen. Abgesehen davon geht es hier auch prinzipiell erst einmal nur darum, die grundsätzlichen Bezüge zwischen System, Norm und Sprechen aufzuzeigen. 
ten ,sprachsozial als partiell systemisch differente Ausschnitte des komplexen Gesamtsystems Einzelsprache“ (2011, S. 51). Daraus können wir schließen: Es ist also prinzipiell möglich, den Systembegriff sowohl auf die Ebene der Einzelsprache zu beziehen als auch auf die Ebene von Varietäten. Wenn man nun davon ausgeht, dass sich das System einer Einzelsprache aus mehreren Systemen von Varietäten zusammensetzt (ausführlicher dazu in Hennig i.Vorb.), lässt sich daraus die Annahme der Rekursivität des Systembegriffs ableiten: Ein System kann aus mehreren (Teil-)Systemen bestehen, die ihrerseits prinzipiell auch wieder aus mehreren (Teil-)Systemen bestehen können. Die folgende Übersicht soll diese Annahme illustrieren:

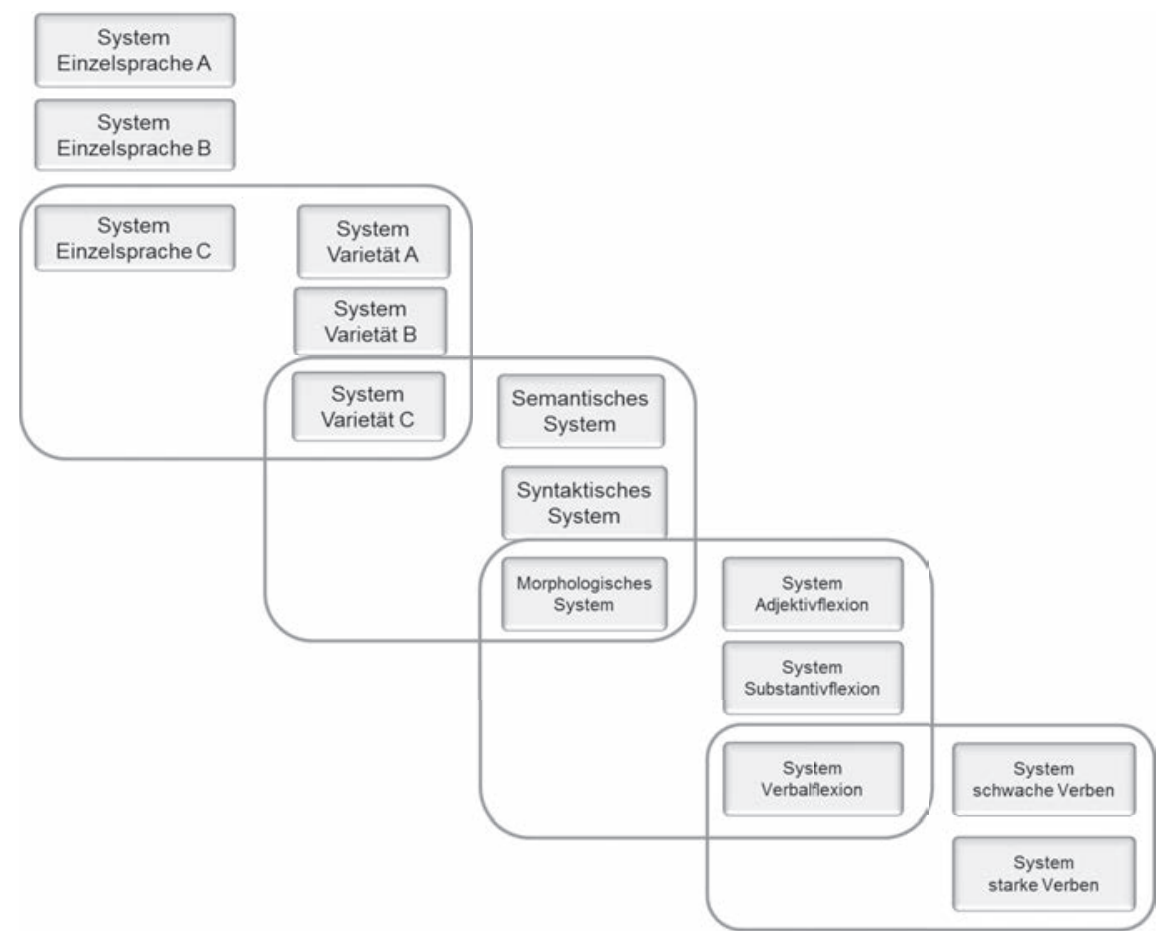

Abb. 1: Rekursivität des Systembegriffs

Dabei handelt es sich um eine vereinfachende Darstellung, die lediglich die Grundidee der Rekursivität des Systembegriffs illustriert. Dabei sind die in der Abbildung angeführten Teilsysteme als rein exemplarisch zu verstehen: So hätten beispielsweise auch einer Einzelsprache A oder B Teilsysteme zugeordnet werden können oder etwa dem syntaktischen System oder dem System der Adjektiv- 
flexion. Einschränkend ist einerseits darauf hinzuweisen, dass die Rekursivität begrenzt ist. Beispielsweise lässt sich in Bezug auf das Beispiel auf der untersten Ebene des rekursiven Modells fragen, ob schwache und starke Verben tatsächlich Teilsysteme des Systems ${ }^{3}$ der Verbflexion bilden, oder ob man hier eher von Klassen sprechen sollte. Andererseits - und das ist eine viel größere Einschränkung - haben die am oberen Ende angegebenen Systemformen ,Einzelsprache ${ }^{6}$ und ,Varietät' einen ganz anderen Status als die im Folgenden aufgeführten auf linguistische Ebenen bezogenen Systemformen. Die Vereinfachung ${ }^{4}$ in der Darstellung soll hier aber in Kauf genommen werden, weil die Annahme der Rekursivität des Systembegriffs für den mittleren Bereich der (Teil-)Systeme linguistischer Ebenen m.E. sehr erhellend für die Beschäftigung mit der Frage nach dem Zusammenhang von System und Variation ist.

Dabei möchte ich auf die Annahme „konfligierender Teilsysteme“ bei Ágel (2008) zurückgreifen. Ágel stellt verschiedene Typen von Grammatikfehlern gegenüber und nimmt dabei einen Typus des Normfehlers an, der auf konfligierende Teilsysteme zurückzuführen ist. Als Beispiele führt er die bereits grammatikalisierte Form frohen Mutes an, die er auf die konfligerenden Teilsysteme der starken und schwachen Adjektivdeklination zurückführt, und den aktuellen Zweifelsfall brauchen mit oder ohne $z u$, der sich aus den konfligierenden Teilsystemen der Modalverben und Halbmodale ergibt. Aus diesen Beispielen lässt sich schlussfolgern: Die Existenz grammatischer Varianten ist in vielen Fällen unmittelbar darauf zurückführbar, dass das System aus Teilsystemen besteht, die unterschiedliche Möglichkeitsräume eröffnen. Da es in vielen Fällen möglich ist, ein Phänomen sowohl auf der Basis der Möglichkeiten eines Teilsystems A als auch eines Teilsystems B zu realisieren, kommt es immer wieder zu Konflikten zwischen Teilsystemen.

3 Ich spreche hier und im Folgenden immer wieder teilweise von ,System“ und teilweise von ,Teilsystem‘. Die Termini sind nicht sauber abgrenzbar, da im Sinne der Rekursivität Systeme, die in Bezug auf ein übergeordnetes System Teilsysteme bilden, wiederum selbst auch wieder aus Teilsystemen bestehen können.

4 Vereinfachend ist an dieser Darstellung darüber hinaus, dass der Eindruck entstehen könnte, dass einzelsprachliche Systeme quasi als reine Additionen verschiedener Systeme von Varietäten bestehen und dass die Varietäten wiederum aus jeweils nur auf diese Varietäten bezogenen Teilsystemen bestehen. Die Zusammenhänge sind allerdings viel komplexer. Man muss sich das Ganze eher kombinatorisch vorstellen: Manche Komponenten von Teilsystemen werden in verschiedenen Varietäten genutzt, teilweise verfügen Varietäten jedoch auch über spezifische systematische Eigenschaften. Ausführlicher dazu in Hennig (i.Vorb.). 
Konfligierende Teilsysteme als Motoren für grammatische Variation können prinzipiell auf verschiedene Ebenen des rekursiven Systembegriffs bezogen werden:

- Konflikte zwischen semantischem und syntaktischem System: Als Beispiel können hier die zahlreichen Fälle an Konflikten zwischen semantischer und grammatischer Kongruenz genannt werden, beispielsweise Das Mädchen sie/es; Ein Kilo Bohnen reichen/reicht aus.

- Konflikte zwischen den Systemen der Adjektivflexion und der Substantivflexion als Teilsysteme des morphologischen Systems der Deklination: Konflikte dieser Art treten bei substantivierten Adjektiven auf, da sich bei diesen die Frage stellt, ob sie dem adjektivischen oder dem substantivischen System folgen sollen: die Aufmachung verschiedener Illustrierter (= stark, adjektivisch)/Illustrierten (= schwach, substantivisch).

- Konflikte zwischen den Systemen der starken und schwachen Flexion: Diese können prinzipiell in allen Bereichen der Unterscheidung zwischen starker und schwacher Flexion beobachtet werden: Substantivflexion: am Automaten/Automat; Adjektivflexion: in gebessertem psychischem/psychischen Zustand; Verbflexion: gewunken/gewinkt; backte/buk.

Bei der Zuordnung von Beispielen konfligierender Teilsysteme zum obigen Modell rekursiver Systeme bestätigt sich, dass die Ebenen der Einzelsprachen und der Varietäten innerhalb des Modells einen völlig anderen Status haben als die unteren aufgeführten Ebenen. Das bedeutet nicht, dass nicht auch Varietäten konfligieren könnten. Aufschlussreich ist diesbezüglich Peter Kochs Ansatz zum Zusammenhang von Variation und Wandel (Koch 2004), nach dem Sprachwandel nicht nur durch die Entstehung neuer sprachlicher Fakten entsteht, „sondern auch eine Veränderung der variationellen Markierung [...] umfasst“ (ebd., S. 606). Der entscheidende Unterschied zwischen Varietätenkonflikten und den hier aufgeführten Konfliktbeispielen auf den unteren Ebenen des Modells besteht darin, dass sich Varietätenkonflikte aus Konflikten verschiedener Teilsysteme speisen. Wenn sich zum Beispiel die Frage stellt, ob und wenn ja unter welchen Bedingungen die in der gesprochenen Sprache übliche Verbzweitstellung nach weil auch in der geschriebenen Sprache Anwendung finden darf, dann handelt es sich um eine Unsicherheit, „deren Quelle die gleichzeitige Beherrschung von mehreren Normen ist, die in verschiedenen Varietäten verankert sind“ (Ágel 2008, S. 68), und die gleichzeitig auf Konflikte zwischen den Systemen der Koordination und Subordination zurückgeht. Konfligierende syntaktische Teilsysteme sind hier also der Gegenstand des Varietätenkonflikts. Daraus folgt aber umgekehrt nicht, dass Konflikte auf den unteren Ebenen des Modells immer auch Gegenstand von Varietätenkonflikten sein müssen. Es ist prinzipiell durchaus möglich, dass auch Teil- 
systeme innerhalb einer Varietät konfligieren. So handelt es sich bei den oben aufgeführten Beispielen zu Konflikten zwischen semantischem und syntaktischem System, dem System der Adjektiv- und Substantivflexion sowie den Systemen der starken und schwachen Flexion aus meiner Sicht um Kandidaten für Konflikte innerhalb des Systems der Standardsprache. Mit der einschränkenden Formulierung ,Kandidaten“ soll aber angedeutet werden, dass es nicht als gesichert angesehen werden kann, ob es tatsächlich eine rein standardinterne Varianz geben kann (vgl. beispielsweise Szczepaniak i.Vorb. zur Erklärung der Genitivvariation durch die Sprache der Nähe vs. Sprache der Distanz).

Als Zwischenfazit lässt sich an dieser Stelle festhalten, dass grammatische Variation einerseits eine funktionale Dimension aufweist, da die Auswahl einer grammatischen Variante in vielen Fällen funktional in Bezug auf verschiedene variationelle Kontexte ist, andererseits aber auch eine sprachsystematische Dimension, indem das Sprachsystem grammatische Variation überhaupt erst ermöglicht. Da grammatische Variation in der Regel im Rahmen der vom System bereitgestellten Möglichkeiten erfolgt, ist sie systematisch. Bestimmte Varianten sind also nicht einfach Abweichungen von einem System X, sondern setzen in der Regel die Existenz von Teilsystemen voraus, in Bezug auf die sie systemkonform sind. Grammatische Varianten befinden sich also nie im luftleeren Raum. Wenn sie dennoch als Abweichung wahrgenommen werden (beispielsweise die Verbzweitstellung nach weil als Abweichung vom System der Standardsprache), dann geschieht dies deshalb, weil sie auf ein System bezogen werden, dem sie gar nicht (bzw. noch nicht oder nicht mehr) angehören. Grammatische Variation ist also höchst systematisch, indem sie sich innerhalb der Möglichkeiten verschiedener (Teil-)Systeme bewegt. Dass sie auf Systemkonflikten aufbaut, macht sie nicht unsystematisch, sondern ist der Komplexität der Beziehungen zwischen verschiedenen Systemebenen geschuldet.

\section{Grammatische Variation als Gegenstand öffentlicher Sprachreflexion}

\subsection{Fragestellung und Datenbasis}

Für den Sprachbenutzer bedeutet die hier skizzierte Komplexität des Systems und die offenbar sehr breite Existenz konfligierender Teilsysteme, dass er ständig Entscheidungen für eine Variante treffen muss. Das hängt u.a. damit zusammen, 
dass zwar die „individuelle Kompetenz [...] zu jedem Zeitpunkt nur einen (jeweils anderen) Ausschnitt der komplexen Gesamtsprache“ umfasst (Schmidt/Herrgen 2011, S. 49), gleichzeitig aber auch mehrere Varietäten:

Es gibt kein Individuum, das überhaupt nichts von anderen Mundarten und Sprachniveaus weiß. Jedes Individuum kennt per definitionem mehrere Sprachstile. Es spricht nicht nur eine einzige funktionelle Sprache innerhalb seiner historischen Sprache. (Coseriu 2007, S. 265)

Der Sprecher muss folglich einerseits stets die Entscheidung für die für seine kommunikativen Absichten jeweils geeignete Varietät und die ihr zuzuordnende Variante treffen, wird aber andererseits aufgrund seines in Bezug auf das komplexe Gesamtsystem immer nur partielle Wissen auch immer wieder mit Phänomenen konfrontiert, die nicht (oder noch nicht) Gegenstand seiner individuellen Sprachkompetenz sind. Dem Phänomen grammatischer Variation kann sich folglich niemand entziehen. Da, wie Schmidt/Herrgen treffend im Rahmen ihrer Definition von ,Varietät‘ erfassen, „Varietäten immer auch sozial konstituiert“ sind (2011, S. 51), hat die Wahrnehmung und Diskussion grammatischer Variationsphänomene in der Gesellschaft einen hohen Stellenwert.

In Abgrenzung von sprachpflegerischen Reaktionen auf die öffentliche Wahrnehmung von Variation hat sich in der germanistischen Linguistik in den letzten Jahren - wegweisend war hier Klein (2003) - die Praxis herausgebildet, den gesellschaftlichen Umgang mit Variation mit dem Terminus ,Zweifelsfall‘ zu erfassen. Zweifelsfälle ergeben sich dann, wenn der kompetente Sprecher aufgrund der Existenz mehrerer Varianten eine Entscheidung treffen muss, wobei es dabei in den meisten Fällen um die Frage geht, welche der Varianten standardsprachlich ist (Klein 2003). Da der Zweifel folglich der alltäglich-natürliche Umgang mit sprachlicher Variation in der öffentlichen Sprachreflexion ist, ergibt sich daraus umgekehrt, dass Zweifelsfälle für die Linguistik eine Art Fenster zu grammatischer Variation bieten.

Der vorliegende Beitrag macht deshalb authentische Zweifelsfälle als Phänomene öffentlicher Sprachreflexion zum empirischen Gegenstand einer Bestandsaufnahme zu grammatischer Variation. Dabei stehen die folgenden Fragen im Mittelpunkt:

1. Welche Bereiche grammatischer Variation sind Gegenstand öffentlicher Sprachreflexion?

2. Welche Arten von grammatischen Variationsphänomenen lassen sich auf welche Arten von variationellen Kontexten beziehen? 
Die auf die jahrzehntelange Sprachberatung der Dudenredaktion zurückgehende lexikographische Praxis der Erfassung grammatischer Variation im Dudenband 9 „Richtiges und gutes Deutsch“ dient als Datengrundlage für die Beschäftigung mit diesen Fragen. ${ }^{5}$ Gegenstand der Analyse war die siebente Auflage (2011). ${ }^{6}$ Die erste Auflage erschien 1965; die lexikographische Praxis der Erfassung von Zweifelsfällen blickt also bereits auf eine fünfzigjährige Tradition zurück. Mit den immerhin knapp 2700 Einzellemmata zu grammatischen Variationsphänomenen liegt uns somit eine recht breite empirische Basis zu grammatischer Variation als Gegenstand öffentlicher Sprachreflexion vor. ${ }^{7}$

Dabei muss einschränkend darauf hingewiesen werden, dass die Auswahl der Phänomene sowohl geprägt ist von der Wörterbuchstruktur des Werks als auch - und vor allem - von den Kontexten, in denen Sprachbenutzer sich an Sprachberatungsstellen wenden. Das sind vorrangig Kontexte der distanzsprachlichen Textproduktion. Peter Eisenberg hat darauf mit seiner Bearbeitung der 6. Auflage 2007 mit dem Konzept der ,geschriebenen Standardsprache als Leitvarietät' reagiert (Näheres dazu in Eisenberg 2007). Ich kann folglich nicht behaupten, mit der Erfassung der in dieses Wörterbuch aufgenommenen Variationsphänomene ein umfassendes Bild grammatischer Variation in sämtlichen Bereichen zu zeichnen, sondern die Bestandsaufnahme bezieht sich vordergründig auf solche Variationsphänomene, die Sprachbenutzer im Kontext distanzsprachlicher Textproduktion zur Nutzung eines Sprachberatungsangebots veranlassen.

\subsection{Grammatische Kernbereiche}

Bei einer ersten Annäherung an das Datenmaterial zeigt sich als auffälliges Ergebnis, dass sich sehr leicht Kernbereiche ausmachen lassen (Tab. 1).

\footnotetext{
5 Dieser Weg wird hier gewählt, weil es bisher keine systematischen Datenbanken zu Sprachberatungsfragen und -antworten gibt. Vereinzelt gibt es jedoch Auswertungen von Sprachberatungsfragen, vgl. Riegel (2007, S. 68 f.) und Seelig (2002).

6 Die Datenerfassung diente auch der Vorbereitung der 2016 erschienenen achten Auflage (Duden 2016).

7 Der Dudenband 9 umfasst insgesamt 5897 Lemmata. Die Datenbasis für diesen Beitrag bilden die 2694 Lemmata zu grammatischen Zweifelsfällen. Für die Erfassung und Auswertung der Daten danke ich Stephanie Lotzow und Melina Möhring.
} 
Tab. 1: Kernbereiche grammatischer Variation im Dudenband 9

\begin{tabular}{llcc}
\hline Themengebiet & Beispiel & Häufigkeit & Prozent \\
\hline Substantiv & Plural von Klotz & 891 & 36,7 \\
\hline Rektion & Rektion der Präposition cif & 481 & 19,8 \\
\hline Verb & Partizip II von schimpfen & 390 & 16,1 \\
\hline Adjektiv & Steigerung von universal & 182 & 7,5 \\
\hline Fugenelement & Fuge Toilette- $n$-... & 120 & 4,9 \\
\hline Kongruenz & Zehn Gramm sind/ist zu wenig & 118 & 4,9 \\
\hline Nominalgruppenflexion & oben genanntem Abgesandten/m & 84 & 3,5 \\
\hline Derivation & Wissenschafter/Wissenschaftler & 59 & 2,4 \\
\hline Attribution & gehuldigt als Attribut & 55 & 2,3 \\
\hline Apposition & ein Glas edler Wein/edlen Weines & 46 & 1,9 \\
\hline Gesamt & & $2426(2694)$ & $90(100)$ \\
\hline
\end{tabular}

Die hier aufgeführten zehn Themengebiete machen 90\% aller Einzellemmata zu grammatischen Themengebieten aus. Die Liste wird angeführt von dem Bereich Substantiv, hierzu gehören Fragen zu den Deklinationsklassen stark/schwach und zu einzelnen Kategorien, insbesondere Plural und Genitiv. Im Bereich Rektion geht es um die Rektion von Verben, Präpositionen, Substantiven und Adjektiven. Bei den Verben spielt ebenfalls die Klassenzugehörigkeit stark/schwach eine große Rolle, aber auch der Bereich der Partikelverbbildung ist prominent vertreten.

Die Top-Ten-Liste deckt sich größtenteils mit vergleichbaren Erfassungen in Hennig (2010) (zu im Rahmen einer Informantenbefragung zur Grammatikbenutzung erfassten grammatischen Fragen) und Hennig/Koch (2016) (zu den Fragen im Sprachberatungsforum www.grammatikfragen.de). Dass der Bereich Substantiv die Liste anführt, ist aber ein Spezifikum der Duden 9-Daten, das sich dadurch erklären lässt, dass diese durch die Wörterbuchstruktur geprägt sind. Zwar kommen in Duden 9 nicht nur einzelne Lexeme als Lemmata in Frage (vgl. dazu Eisenberg 2007, S. 210 f.), dennoch eignen sich lexembezogene Zweifelsfälle prinzipiell besser für die lexikographische Erfassung als etwa syntagmatisch komplexe Phänomene wie Kongruenz und Nominalgruppenflexion, die in den anderen genannten Erfassungen vor Substantiv stehen.

Wenn man die Ergebnisse auf das in Kapitel 3 vorgestellte Modell des rekursiven Systembegriffs bezieht, so ist festzustellen, dass sich die Wahrnehmung von grammatischer Variation in der öffentlichen Sprachreflexion auf die unteren Ebe- 
nen des Modells konzentriert. Dabei kann die Annahme konfligierender Teilsysteme als erklärungsrelevant betrachtet werden. So lassen sich die Variationsphänomene der Kernbereiche problemlos auf konfligierende Teilsysteme bzw. Klassen zurückführen. Zentrale Phänomene/Konflikte sind:

\section{Substantiv}

- starke vs. schwache Deklination

- verschiedene Möglichkeiten der Pluralbildung

- verschiedene Möglichkeiten der Genuszuweisung

Verb

- starke vs. schwache Konjugation

- Partikelverb vs. Präfixverb

Rektion

- Rektion verschiedener Kasus

- Rektion verschiedener Präpositionen

- Rektion von Kasus vs. Präposition

Nominalgruppenflexion

- starke vs. schwache Deklination des Adjektivs

- Parallel- vs. Wechselflexion von aufeinander folgenden Adjektiven

- pronominale vs. adjektivische Flexion spezieller Artikelwörter

\subsection{Zusammenhänge zwischen systematischer und funktionaler Variation}

Nach dem allgemeinen Überblick über Variationsphänomene in Sprachberatungskontexten soll es nun um die Frage gehen, welche Aussagen zur variationslinguistischen Verortung der in Duden 9 erfassten Phänomene grammatischer Variation getroffen werden können. Es geht also um die Frage, ob sich einzelne Variationsphänomene systematisch bestimmten funktionalen Variationsbereichen zuordnen lassen. Um dieser Frage nachgehen zu können, wurden zu den Einzellemmata von Duden 9 auch die dort verwendeten Varianzausdrücke erfasst. Das Konzept der Varianzausdrücke geht auf eine frühere Arbeit zur Dudengrammatik zurück (Hennig/Hochscheid/Löber 2012), da sich in der Dudengrammatik ebenso wie in Duden 9 die Praxis etabliert hat, dem Präskriptionsbedürfnis vieler Nutzer durch möglichst genaue Angaben zum Sprachgebrauch zu begegnen. Als Varianzausdrücke wurden in der genannten Studie solche sprachlichen Ausdrücke bezeichnet, mit denen die Gültigkeit einer Aussage eingeschränkt wird, indem sie 
auf einen bestimmten Bereich festgelegt wird. Unter dieses recht allgemeine Verständnis von ,Varianzausdruck' lassen sich einerseits diasystematische und diachronische Ausdrücke einordnen, andererseits auch Frequenzangaben. Neben einer Einordnung von grammatischen Varianten mit Hilfe von Ausdrücken der genannten Art kann auch eine Bewertung der Varianten mit Hilfe von präskriptiven Ausdrücken erfolgen. Dieser Bereich wird hier relativ weit gefasst: Er umfasst sowohl Negativ- als auch Positivbewertungen, also im Grunde alle Einordnungen, die über diasystematische und diachrone Ausdrücke sowie Frequenzausdrücke hinausgehen. ${ }^{8}$

Für die folgende Analyse der Einordnung von Variationsphänomenen in Duden 9 ergeben sich die folgenden Analysekategorien:

- Vorhandensein eines präskriptiven Ausdrucks (z.B. fälschlicherweise, besser, durchaus akzeptabel, noch nicht zulässig, stilistisch unschön, ist vorzuziehen);

- Vorhandensein eines Frequenzausdrucks (z.B. häufig, selten, fast ausschließlich, im Allgemeinen, kommt auch vor);

- Vorhandensein eines diachronen Ausdrucks (z.B. früher gebräuchlich, heute nur noch, immer seltener, veraltend);

- Vorhandensein eines diasystematischen Ausdrucks:

- diamedial (z.B. geschriebene Sprache, gesprochene Sprache),

- diaphasisch (z.B. Amtssprache, Bergmannssprache, Ingenieurwesen, Überseehandel),

- diatopisch (z.B. österreichisch, Süd- und Westdeutschland, oberdeutsch),

- diastratisch (z.B. gehoben, gewählte Sprache, saloppe Redensweise),

- Basiskategorien (z.B. Standardsprache, Umgangssprache, Gemeinsprache).

Der Bereich diasystematischer Ausdrücke wurde im Wesentlichen auf der Basis der Grundkategorien diaphasisch, diatopisch und diastratisch sowie (in Anlehnung an Koch/Oesterreicher 1994) diamedial erfasst. Das ist natürlich ein sehr grobes Raster, das an vielen Stellen Fragen aufwirft (gehört beispielsweise Literatursprache zur Diaphasik oder zur Diastratik? Wie unterscheidet man also so etwas wie Situationsbezogenheit und Gruppenzugehörigkeit? Wie isoliert man

8 Unter ,Präskription“ werden hier also alle Versuche des unmittelbaren Eingriffs in den Sprachgebrauch verstanden. Natürlich können - das wissen wir spätestens seit Klein (2004) - auch deskriptiv gemeinte Kategorien vom Rezipienten präskriptiv verstanden werden. Als präskriptive Ausdrücke werden hier deshalb nur solche Ausdrücke verstanden, mit denen autorseitig bereits eine Überschreitung der reinen Deskription vorgenommen wird, mit denen der Autor sich also - ganz im Sinne der Ratgeberkonzeption - um eine Empfehlung bemüht. Hilfreich ist in diesem Kontext der Begriff des ,Präskriptionspotenzials‘, mit dem Lotzow (2016) in ihrer Nutzerstudie zu Duden 9 das Potenzial von bestimmten Kategorien zur Beeinflussung einer Entscheidung für eine Variante beschreibt. 
überhaupt - angesichts der sehr nachvollziehbaren Annahme von Varietätenketten (Koch/Oesterreicher 1994, S. 595) - einzelne Diadimensionen?). Im Sinne einer an die Forschungstradition anschlussfähigen Erfassungsheuristik kann das aber m.E. in Kauf genommen werden. Lediglich Markierungen wie ,standardsprachlich“ und ,umgangssprachlich“ wurden hier zusätzlich als ,Basiskategorien' erfasst, weil sie gewissermaßen quer zu den Diadimensionen liegen. Nicht unerwähnt soll schließlich bleiben, dass die Zuordnung einzelner Varianzausdrücke zu den vorgestellten Dimensionen teilweise auch deshalb problematisch ist, weil sie nicht immer genau einer Analysekategorie zugeordnet werden können, sondern häufig auch fusionierend sind. So enthält ein Ausdruck wie üblich sowohl Aspekte von Frequenz (vergleichbar mit oft, häufig), aber auch von Normbezogenheit (normal).

Welch große Rolle die Varianzausdrücke insgesamt im Dudenband 9 spielen, soll die folgende Übersicht zu den Zahlen der ermittelten Varianzausdrücke in den Einzellemmata zu grammatischen Phänomenen illustrieren:

Tab. 2: Typen von Varianzausdrücken im Dudenband 9 (type-Frequenz)

\begin{tabular}{lcc}
\hline Varianzausdruck (type) & absolut & prozentual (Anteil an types) \\
\hline diachron & 81 & $20,4 \%$ \\
\hline diamedial & 5 & $1,3 \%$ \\
\hline diaphasisch & 59 & $14,8 \%$ \\
\hline diastratisch & 11 & $2,8 \%$ \\
\hline diatopisch & 33 & $8,3 \%$ \\
\hline Basiskategorie & 11 & $2,8 \%$ \\
\hline Frequenzausdruck & 108 & $27,1 \%$ \\
\hline Präskriptiver Ausdruck & 90 & $22,6 \%$ \\
\hline Gesamt & 398 & $100 \%$ \\
\hline
\end{tabular}

Dabei handelt es sich zunächst ausschließlich um Angaben zu types. Die hohe Anzahl an Typen von diaphasischen Ausdrücken beispielsweise kommt dadurch zustande, dass der Bereich der Fachsprachen sehr stark ausdifferenziert ist, er reicht von allgemeinen Kategorien wie ,Fachsprache“ oder ,Amtsdeutsch“ bis hin zu sehr spezifischen Kategorien wie ,Militär‘, ,Textilwesen', und ,katholische Kirche‘. Welche Rolle die Varianzausdrücke im Sinne einer Tokenfrequenz spielen, ist Gegenstand der folgenden Auswertungen. 


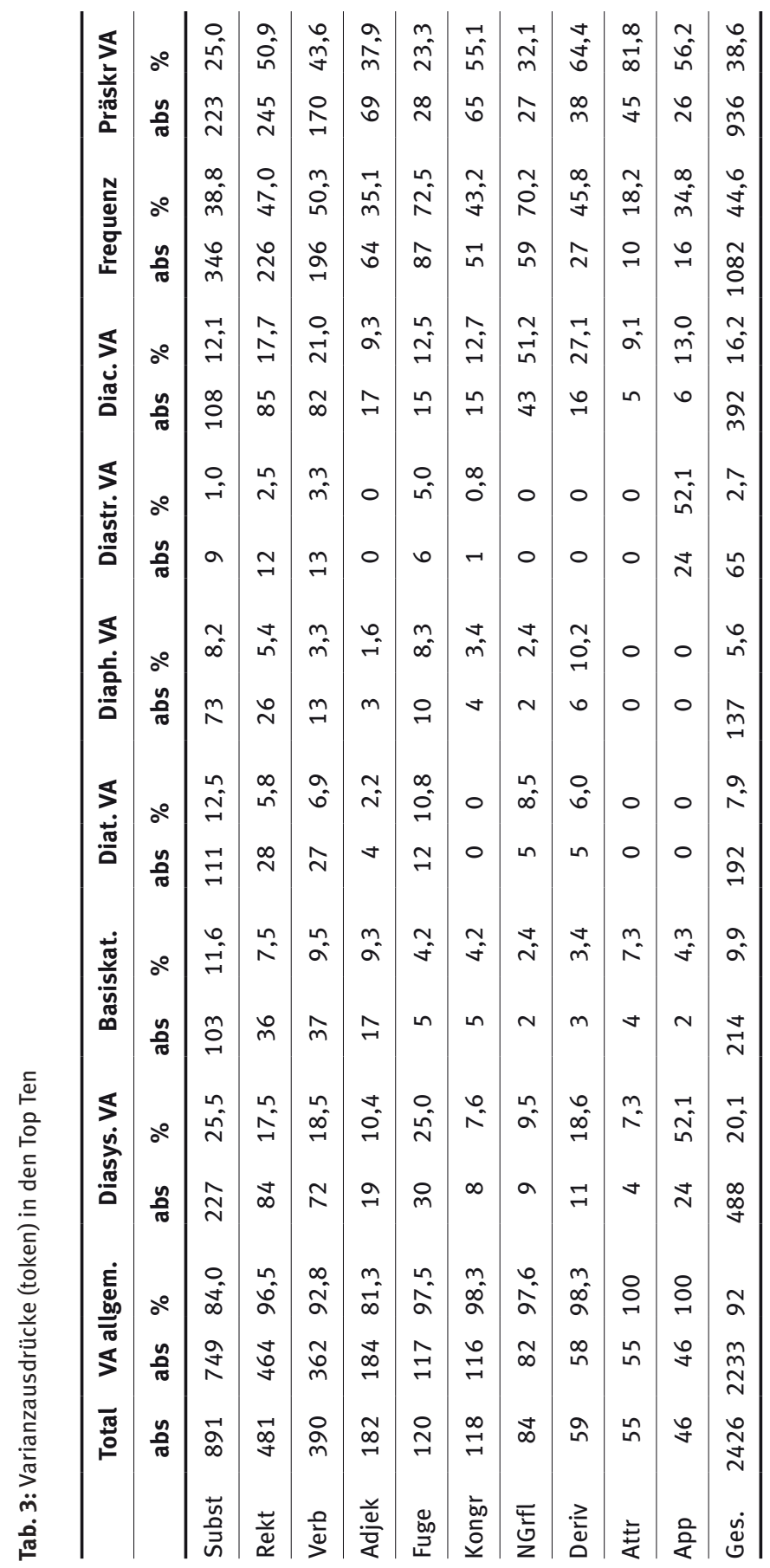


Die Angaben sind folgendermaßen zu lesen: Die erste Spalte führt die Gesamtanzahl der Lemmata der jeweiligen Themengebiete als Vergleichsbasis auf. Die Prozentzahlen zu den Varianzausdrücken beziehen sich jeweils auf diese Gesamtzahl. Beispielsweise enthalten von den 891 Lemmata zu Substantiven 749 einen Varianzausdruck, das entspricht einem Anteil von 84\%. Die Prozentzahlen in der untersten Zeile „Gesamt“ hingegen beziehen sich auf die Gesamtzahl der TopTen-Lemmata (2426). Hier sieht man, wie hoch der Anteil der Lemmata mit einem Varianzausdruck der genannten Art an den Lemmata gesamt ist. Dass sich in dieser Zeile insgesamt nicht 100 Prozent ergeben, liegt daran, dass in einem Lemma mehrere Varianzausdrücke vorhanden sein können. Es ergeben sich die folgenden Eindrücke zu den einzelnen Bereichen:

- Varianzausdrücke allgemein: Der Tabelle ist zu entnehmen, dass die Varianzausdrücke insgesamt eine sehr große Rolle in Duden 9 spielen. Man bemüht sich also darum, mit Hilfe solcher Ausdrücke Variation systematisch zu erfassen. Dabei kehrt sich die Top-Ten-Hierarchie hier quasi um, die niedrigfrequenten Bereiche werden also flächendeckender mit Varianzausdrücken eingeordnet als die hochfrequenten.

- Diasystematische Ausdrücke allgemein: Aus der insgesamt niedrigen Gesamtzahl an diasystematischen Varianzausdrücken im Vergleich zur Gesamtanzahl sowie zu den präskriptiven Ausdrücken und den Frequenzausdrücken kann geschlossen werden, dass die Einordnung in diasystematische Zusammenhänge bei vielen Phänomenen - etwa im Bereich Adjektiv, Nominalgruppenflexion, Kongruenz, Attribution - eine untergeordnete Rolle spielt.

- Basiskategorien: Wenn man dabei bedenkt, wie wichtig das Konzept der „geschriebenen Standardsprache als Leitvarietät“ seit 2007 für den Duden 9 ist (vgl. Eisenberg 2007), ist der Anteil an Basiskategorien insgesamt nicht sehr hoch. Das kann wohl damit erklärt werden, dass die ,geschriebene Standardsprache' gerade weil sie Leitvarietät ist, nicht immer explizit benannt werden muss. Hier gilt wohl: Wenn keine gegenteilige Markierung vorgenommen wurde (beispielsweise die Zuordnung zu einer spezifischen Varietät oder ein präskriptiver Ausdruck), kann die Variante (insbesondere dann, wenn sie mit der Dudenempfehlung als präferierte Variante gekennzeichnet ist) als der geschriebenen Standardsprache angehörig betrachtet werden. Beispiele für Kennzeichnungen mit Basiskategorien sind die Einordnung der Pluralform Klötze als standardsprachlich und der Pluralform Klötzer als umgangssprachlich sowie das regional verbreitete Partizip II geschumpfen von schimpfen als nicht standardsprachlich.

- Diatopik: Die insgesamt niedrige Anzahl an diatopischen Ausdrücken zeigt, dass die regionalsprachliche Verortung insgesamt keine sehr große Rolle in Duden 9 spielt. Auch von Dürscheid/Sutter wird der Eingang der gramma- 
tisch-diatopischen Variation in das Wörterbuch als „marginal“ bezeichnet (2014, S. 53). Dürscheid/Elspaß (2015) konstatieren außerdem, dass die Ausführungen zu grammatischen Phänomenen am norddeutschen Gebrauchsstandard ausgerichtet seien.

- Diaphasik: Im Vergleich zur hohen type-Frequenz ist die token-Frequenz im Bereich der diaphasischen Ausdrücke recht niedrig. Folglich findet in diesem Bereich - wie oben bereits angemerkt - eine sehr hohe Ausdifferenzierung statt, viele diaphasische Ausdrücke kommen also nur einmal oder nur sehr selten vor. Beispiele sind die Zuordnung der Präposition cif mit Akkusativ zum Überseehandel oder auch das maskuline Genus für Patriarchat im kirchlichen Sprachgebrauch.

- Diastratik: Dass der Bereich der Apposition hier im Vergleich zu den insgesamt niedrigen Zahlen so stark vertreten ist, liegt daran, dass es sich bei den als ,gehoben' gekennzeichneten Fällen um einen immer wiederkehrenden Typus von Varianz handelt, und zwar um die Varianten partitive Apposition vs. partitiver Genitiv nach Maß-, Behälter und Mengenangaben. Beispiele sind: Ein Glas edler Wein/edlen Weines, eine Kanne duftender Kaffee/duftenden Kaffees, ein Seidel dunkles Bier/dunklen Bieres. Dabei wird jeweils die Genitivvariante als ,gehoben' gekennzeichnet.

- Diachronie: Im Vergleich zu einigen diasystematischen Bereichen spielen diachrone Ausdrücke insgesamt eine größere Rolle, als man erwarten könnte: Die Notwendigkeit der Verwendung diachroner Ausdrücke ergibt sich teilweise, wenn auf ältere literarische Beispiele zurückgegriffen wird. Aber auch außerhalb dieser Kontexte finden sich häufig Ausdrücke wie ,veraltend‘. Im Bereich der Nominalgruppenflexion geht die Dudenredaktion offenbar davon aus, dass bei substantivierten Adjektiven nach einem weiteren Adjektiv die starke Flexion (also Parallelflexion) sich durchgesetzt hat und die schwache Flexion entsprechend veraltend sei.

- Frequenz: Frequenzausdrücke kommen insgesamt deutlich häufiger vor als diasystematische Ausdrücke. Dabei ist hier einerseits nicht hinreichend klar, wie zwischen den einzelnen Ausdrücken differenziert wird (was ist beispielsweise der Unterschied zwischen oft und häufig oder gelegentlich und manchmal? Vgl. dazu Hennig/Hochscheid/Löber 2012). Andererseits fehlt offenbar in vielen Fällen eine klare korpuslinguistische Absicherung (vgl. dazu Eber/ Rössler 2016). Aus der insgesamt höheren Anzahl von Frequenzausdrücken gegenüber diasystematischen Ausdrücken ergibt sich automatisch, dass diese auch ohne Angaben zur diasystematischen Verortung vorkommen können. So finden sich insgesamt 887 Belege für Frequenzausdrücke ohne diasystematische Markierung. Das ist deshalb relevant, weil es ein Indiz dafür ist, dass tatsächlich ein nicht unerheblicher Teil der Phänomene - etwa ein Vier- 
tel aller grammatischen Einzellemmata - möglicherweise eine rein varietätenintern bedingte Variabilität aufweisen. Es handelt sich dann also um Bereiche, in denen konfligierende Teilsysteme nicht mit Varietätenkonflikten korrelieren.

- Präskription: Dass viele präskriptive Varianzausdrücke vorhanden sind, liegt einerseits an dem weiten Verständnis von Präskription, das hier angewendet wurde. Andererseits hängt es natürlich auch damit zusammen, dass sich der Dudenband 9 trotz der seit der Bearbeitung durch Peter Eisenberg zu beobachtenden Bemühungen um Präskriptionsabbau nach wie vor als Ratgeber versteht, der den Nutzern eine schnelle Entscheidungshilfe bieten möchte. Dass der Bereich der Attribution hier besonders stark vertreten ist, kann dadurch erklärt werden, dass häufig Aussagen dazu getroffen werden, welche Partizipien nicht attributiv verwendet werden dürfen, z.B. ist es laut Duden 9 nicht zulässig zu sagen der gehuldigte Fürst.

Die folgende Übersicht fasst die Ergebnisse zusammen:

\begin{tabular}{|l|l|l|l|l|l|l|l|}
\hline $\begin{array}{l}\text { Varianz- } \\
\text { ausdruck } \\
\text { allgemein }\end{array}$ & $\begin{array}{l}\text { Diasystema- } \\
\text { tik allgemein }\end{array}$ & $\begin{array}{l}\text { Basis- } \\
\text { kategorien }\end{array}$ & Diatopik & Diaphasik & Diastratik & $\begin{array}{l}\text { Frequenz- } \\
\text { ausdruck }\end{array}$ & $\begin{array}{l}\text { Präskriptive } \\
\text { Ausdrücke }\end{array}$ \\
\hline $\begin{array}{l}\text { Attribution } \\
\text { Apposition }\end{array}$ & $\begin{array}{l}\text { Apposition } \\
\text { Substantiv }\end{array}$ & $\begin{array}{l}\text { Substantiv } \\
\text { Verb }\end{array}$ & $\begin{array}{l}\text { Substantiv } \\
\text { Fugenelem. }\end{array}$ & $\begin{array}{l}\text { Derivation } \\
\text { Fugenelem. }\end{array}$ & $\begin{array}{l}\text { Apposition } \\
\text { Fugenelem. }\end{array}$ & $\begin{array}{l}\text { Fugenelem. } \\
\text { NGrflexion }\end{array}$ & $\begin{array}{l}\text { Attribution } \\
\text { Derivation }\end{array}$ \\
\hline $\begin{array}{l}\text { Substantiv } \\
\text { Adjektiv }\end{array}$ & $\begin{array}{l}\text { Kongruenz } \\
\text { Attribution }\end{array}$ & $\begin{array}{l}\text { Derivation } \\
\text { NGrflexion }\end{array}$ & $\begin{array}{l}\text { Attribution } \\
\text { Appostion }\end{array}$ & $\begin{array}{l}\text { Attribution } \\
\text { Apposition }\end{array}$ & $\begin{array}{l}\text { Derivation } \\
\text { Attribution }\end{array}$ & $\begin{array}{l}\text { Apposition } \\
\text { Attribution }\end{array}$ & $\begin{array}{l}\text { Substantiv } \\
\text { Fugenelem. }\end{array}$ \\
\hline
\end{tabular}

Abb. 2: Zusammenfassung der Ergebnisse

Oben sind hier jeweils die beiden Bereiche angeführt, die am häufigsten mit Hilfe eines entsprechenden Varianzausdrucks eingeordnet wurden, unten die beiden, bei denen das am seltensten der Fall war. Es lassen sich die folgenden Tendenzen ableiten:

- bei ,Attribution“ gibt es einen systematischen Zusammenhang zwischen seltener diasystematischer Markierung und Frequenzangaben einerseits und der häufigen Verwendung von präskriptiven Ausdrücken andererseits;

- bei ,Apposition“ gibt es insgesamt ähnliche Tendenzen wie bei ,Attribution“, aber hier gibt es darüber hinaus die mit Abstand häufigste diastratische Markierung; 
- bei ,Substantiv` zeigt sich, dass ein Thema gleichzeitig Spitzenreiter bestimmter Varianzbereiche und (fast) Schlusslicht bei Varianzausdrücken allgemein sein kann (der Grund ist wahrscheinlich die hohe Frequenz insgesamt);

- ,Fugenelement ' ist insgesamt offenbar ein günstiger Kandidat für diasystematische Markierungen und Frequenzaussagen.

Dabei muss an dieser Stelle ausdrücklich betont werden, dass diese Ergebnisse eher den Status von Hypothesen als von uneingeschränkt zuverlässigen, gar statistisch signifikanten Ergebnissen haben. Die Ergebnisse sind zunächst einmal Ergebnisse zum Umgang mit grammatischer Variation in Duden 9. Als solche sind sie einerseits geprägt von der Wörterbuchstruktur, andererseits muss wohl auch davon ausgegangen werden, dass nicht alle Einordnungen korpuslinguistisch abgesichert sind. Die hier vorgestellten Ergebnisse können deshalb nicht den Anspruch erheben, tatsächlich grammatische Variation im Sprachgebrauch insgesamt abzubilden, einige Tendenzen zeigen sie wohl dennoch auf.

\subsection{Die Nutzerperspektive: Zur Wahrnehmung von Varianzausdrücken}

Abschließend sei kurz auf die Nutzerstudie von Lotzow (2016) eingegangen, da dieser Studie Ergebnisse zu der Frage entnommen werden können, wie eigentlich der Erwartungshorizont der Nutzer in Bezug auf den Umgang mit grammatischer Variation im Zweifelsfälleduden aussieht. An der Studie haben 250 Personen teilgenommen, von denen 150 angaben, den Dudenband 9 regelmäßig zu benutzen. Den Teilnehmern wurden exemplarische Zweifelsfälle vorgelegt. Sie wurden erst darum gebeten, eine Entscheidung auf der Basis ihres Sprachgefühls zu treffen, anschließend wurde ihnen die entsprechende Textstelle aus Duden 9 vorgelegt mit der Aufforderung, nach der Lektüre eine endgültige Entscheidung zu treffen.

Bei der Frage danach, wie hilfreich einzelne Gruppen von Varianzausdrücken bei der Entscheidung insgesamt gewesen sind (,Ja bedeutet hier also: „war hilfreich“), ergab sich das interessante Ergebnis, dass die diasystematischen Ausdrücke am besten abgeschnitten haben, gefolgt von diachronen Ausdrücken und eindeutigen Frequenzangaben. ${ }^{9}$ Präskriptive Ausdrücke und unspezifische Frequenzangaben hingegen wurden von den Nutzern als weniger hilfreich empfunden:

9 Als ,eindeutig، wurden hier Frequenzangaben wie selten, meist, häufig eingeordnet, als uneindeutig Frequenzangaben wie im Allgemeinen, gewöhnlich. Genaueres dazu in Hennig/ Hochscheid/Löber (2012). 


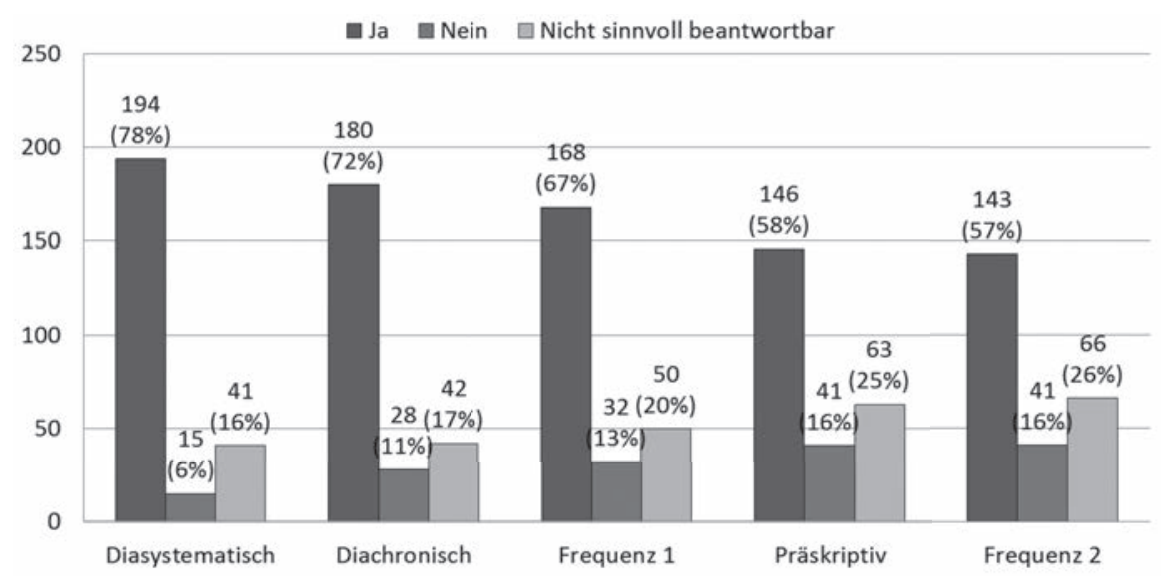

Abb. 3: Bewertung des Nutzens einzelner Typen von Varianzausdrücken durch Duden 9-Nutzer (Lotzow 2016, S. 20)

Daraus können wir schlussfolgern: Die Öffentlichkeit erwartet von uns in erster Linie genaue Angaben zu den Verwendungskontexten.

\section{Schluss}

Das zentrale Anliegen des vorliegenden Beitrags bestand darin, systematische Bezüge zwischen System und Variation theoretisch zu modellieren und durch Daten zu von der Öffentlichkeit wahrgenommenen Variationsphänomenen empirisch zu unterfüttern. Mit der Annahme der Rekursivität des Systembegriffs wurde ein Verständnis von ,Sprachsystem 'vorgestellt, das das Sprachsystem einer Einzelsprache als komplexes Gebilde verschiedener Teilsysteme betrachtet, die im Sinne eines Baukastenprinzips (Hennig i.Vorb.) kombinatorisch in verschiedenen variationell-funktionalen Bereichen zum Tragen kommen. Mit einem solchen dynamischen Verständnis von Sprachsystem wird das System als der Möglichkeitsrahmen verstanden, in dem Variation stattfinden kann. Grammatische Variation wird in diesem Sinne nicht einfach auf die Ebene der Norm ausgelagert oder gar als Abweichung vom System verstanden, sondern als notwendige Folge der Komplexität des Systems: Da das Sprachsystem einer Einzelsprache aus verschiedenen Teilsystemen mit ihren jeweils eigenständigen Gesetzmäßigkeiten besteht, ist es wohl nicht vermeidbar, dass die verschiedenen Gesetzmäßigkeiten der verschiedenen Teilsysteme in Konflikt geraten können. Dadurch entsteht in vielen 
Fällen die Möglichkeit, ein sprachliches Phänomen A entsprechend eines Teilsystems (oder einer Klasse) X systemkonform zu realisieren oder entsprechend eines Teilsystems (oder einer Klasse) Y. Die Variationsmöglichkeiten ergeben sich folglich dadurch, dass teilweise verschiedene Teilsysteme den Möglichkeitsrahmen für die Realisierung eines Phänomens bereitstellen, sodass es dazu kommen kann, dass Teilsystem A eine Variante A ermöglicht und Teilsystem B eine Variante B. Die empirischen Befunde haben ergeben, dass das auf diese Weise entstehende Potenzial des Aufeinandertreffens konfligierender Teilsysteme beträchtlich ist. Zumindest die hier ausgewerteten Daten zu von der Öffentlichkeit wahrgenommenen Phänomenen grammatischer Variation haben den Eindruck entstehen lassen, dass der Ansatz ein hohes Erklärungspotenzial in Bezug auf grammatische Variation hat.

Dabei ist es unvermeidlich, dass Fragen offenbleiben. Die interessanteste dieser Fragen könnte die Frage danach sein, warum es überhaupt konfligierende Teilsysteme im hier beschriebenen Sinne gibt, d.h., warum es sozusagen keine grundlegenden Reparaturmechanismen innerhalb des Systems einer Einzelsprache gibt, die dafür sorgen, dass sich die einzelnen Systembausteine nicht in die Quere kommen. Wahrscheinlich hängt das u.a. damit zusammen, dass es kein übergeordnetes Grundprinzip gibt, das grundlegend regelt, welche vom System bereitgestellten Möglichkeiten im Sprachgebrauch gegenüber anderen bevorzugt werden. So zeigt sich beispielsweise im Bereich der Verbflexion eine große Beharrlichkeit der wenigen starken Verben, obwohl es im Sinne eines Ökonomieprinzips einerseits ökonomischer wäre, nur eine Flexionsvariante $\mathrm{zu}$ haben und andererseits die schwache Verbflexion aufgrund der größeren Einheitlichkeit der Flexionsprinzipien ökonomischer ist als die starke. Offenbar kommt dem Faktor Ökonomie hier der Faktor Frequenz in die Quere, d.h., die starke Verbflexion trotzt dem Ökonomieprinzip auf der Basis der Vorkommenshäufigkeit der entsprechenden Verblexeme. Andere Beispiele zeigen wiederum, dass grammatische Varianten ihre Existenz bzw. das Andauern ihrer Existenz auch ihrem gesellschaftlichsozialen Prestige verdanken können (z.B. die Genitivrektion). Kurz: Wie diese und andere Faktoren zusammenwirken, ist eine spannende Frage, die der vorliegende Beitrag nicht beantworten kann.

Der Beitrag kann darüber hinaus nur als eine erste Annäherung an die Frage betrachtet werden, ob es möglicherweise systematische Zusammenhänge zwischen der Verortung von Variationsphänomenen im System und ihrer funktionalen Erklärung gibt, m.a.W., ob bestimmte Typen von grammatischen Varianten systematisch mit verschiedenen variationell-funktionellen Kontexten korrelieren. Es handelt sich hier deshalb nur um eine erste Annäherung, weil die lexikographischen Daten nicht wie Korpusdaten unmittelbare Sprachgebrauchsdaten sind. Außerdem können die Zuordnungen von Variationsphänomenen zu diasystema- 
tischen Bereichen sowie ihre Einordnung mit Frequenzangaben in Duden 9 nur bedingt als Abbilder des Sprachgebrauchs betrachtet werden, weil nicht davon ausgegangen werden kann, dass diese Einordnungen lückenlos korpuslinguistisch abgesichert sind. Andererseits zeigt gerade der Versuch der Dudenredaktion, grammatische Variationsphänomene möglichst flächendeckend variationslinguistisch einzuordnen, dass ein allgemeiner Bedarf daran besteht, im Sprachgebrauch wahrgenommene formale Varianten funktional auf verschiedene Verwendungskontexte zu beziehen. Dieser Bedarf bestätigte sich auch durch die Ergebnisse der Nutzerstudie von Lotzow (2016). Diese Ergebnisse sprechen dafür, dass es sinnvoll sein könnte, Versuche zu einer die üblichen Grenzen zwischen Teildisziplinen überschreitenden integrativen Auseinandersetzung mit dem Phänomen der grammatischen Variation auszubauen: Wir können unserer Bringschuld im Bereich grammatischer Variation nur gerecht werden, wenn wir den aktuellen Tendenzen zu einer starken Ausdifferenzierung linguistischer Teildisziplinen zum Trotz immer wieder mal den Blick über den Tellerrand wagen.

\section{Literatur}

Ágel, Vilmos (2008): Bastian Sick und die Grammatik. Ein ungleiches Duell. In: InfoDaF, S. 64-84. Coseriu, Eugenio (1971): Sprache. Strukturen und Funktionen. 12 Aufsätze zur allgemeinen und romanischen Sprachwissenschaft. 2., verb. Aufl. (= Tübinger Beiträge zur Linguistik 2). Tübingen.

Coseriu, Eugenio (2007): Sprachkompetenz. Grundzüge der Theorie des Sprechens. Bearb. und hrsg. von Heinrich Weber. 2., durchges. Aufl. (= Tübinger Beiträge zur Linguistik 508). Tübingen.

Dudengrammatik = Duden (2016): Der Duden in 12 Bänden. Bd. 4: Die Grammatik. Unentbehrlich für richtiges Deutsch. 9., vollst. überarb. und aktual. Aufl. Berlin.

Duden 9 = Duden (2011): Der Duden in 12 Bänden. Bd. 9: Richtiges und gutes Deutsch. Das Wörterbuch der sprachlichen Zweifelsfälle. 7., vollst. überarb. Aufl. Mannheim u.a.

Duden (2016): Der Duden in 12 Bänden. Bd. 9: Richtiges und gutes Deutsch. Wörterbuch der sprachlichen Zweifelsfälle. 8., vollst. überarb. Aufl. Hrsg. v. Mathilde Hennig. Berlin: Dudenverlag.

Durrell, Martin (2004): Linguistic variable - linguistic variant. In: Ammon, Ulrich et al. (Hg.): Soziolinguistik. 1. Halbbd. 2., vollst. überarb. und erw. Aufl. (= Handbücher zur Sprachund Kommunikationswissenschaft 3.1). Berlin, S. 195-200.

Dürscheid, Christa/Elspaß, Stephan (2015): Variantengrammatik des Standarddeutschen. In: Kehrein, Roland/Lameli, Alfred/Rabanus, Stefan (Hg.): Regionale Variation des Deutschen. Projekte und Perspektiven. Berlin/Boston, S. 563-584.

Dürscheid, Christa/Sutter, Patrizia (2014): Grammatische Helvetismen im Wörterbuch. In: Zeitschrift für Angewandte Linguistik 60, S. 37-65. 
Eber, Franziska/Rössler, Paul (2016): Modalisierte Assertionen in Kodizes. Zu Formulierungsstrategien im Duden-Band 9 „Richtiges und gutes Deutsch“. In: Klein/Staffeldt (Hg.), S. $149-161$.

Eisenberg, Peter (2007): Sprachliches Wissen im Wörterbuch der Zweifelsfälle. Über die Rekonstruktion einer Gebrauchsnorm. In: Aptum. Zeitschrift für Sprachkritik und Sprachkultur 3, S. 209-228.

Fuß, Eric/Wöllstein, Angelika (Hg.) (i.Vorb.): Ars grammatica. Grammatiktheorie und Grammatikographie. (= Studien zur Deutschen Sprache). Tübingen.

Hennig, Mathilde (2010): Plädoyer für eine Grammatikbenutzungsforschung: Anliegen, Daten, Perspektiven. In: Deutsche Sprache 38, S. 19-42.

Hennig, Mathilde (i.Vorb.): Grammatik und Variation in Grammatikforschung und Grammatikschreibung. In: Fuß/Wöllstein (Hg.).

Hennig, Mathilde/Hochscheid, Thomas/Löber, Melanie (2012): Sind Varianzausdrücke ein geeignetes Mittel zur Bewältigung des Normativitätsdilemmas? In: Zeitschrift für Angewandte Linguistik 56, S. 95-120.

Hennig, Mathilde/Koch, Dennis (2016): Zum Verhältnis von Sprachberatung und Kodifizierung. Das Beispiel www.grammatikfragen.de. In: Klein/Staffeldt (Hg.), S. 70-84.

Klein, Wolf Peter (2003): Sprachliche Zweifelsfälle als linguistischer Gegenstand. Zur Einführung in ein vergessenes Thema der Sprachwissenschaft. In: Linguistik online 16. Internet: www.linguistik-online.de/16_03/klein.html (Stand: 4.8.2016).

Klein, Wolf Peter (2004): Deskriptive statt präskriptiver Sprachwissenschaft!? In: Zeitschrift für germanistische Linguistik 32, S. 376-405.

Klein, Wolf Peter/Staffeldt, Sven (Hg.) (2016): Die Kodifizierung der Sprache. Strukturen, Funktionen, Konsequenzen. (= WespA - Würzburger elektronische sprachwissenschaftliche Arbeiten 17). Würzburg.

Koch, Peter (2004): Sprachwandel, Mündlichkeit und Schriftlichkeit. In: Zeitschrift für romanische Philologie, S. 605-630.

Koch, Peter/Oesterreicher, Wulf (1994): Schriftlichkeit und Sprache. In: Günther, Hartmut/ Ludwig, Otto (Hg.): Schrift und Schriftlichkeit. 1. Halbbd. (= Handbücher zur Sprach- und Kommunikationswissenschaft 10.1). Berlin/New York, S. 587-604.

Konopka, Marek/Fuß, Eric (2016): Genitiv im Korpus. Pilotuntersuchungen zur starken Flexion des Nomens im Deutschen. (= Studien zur Deutschen Sprache 70). Tübingen.

Lotzow, Stephanie (2016): Rezeptionsorientierter Umgang mit Varianzausdrücken bei grammatischen Zweifelsfällen. In: Klein/Staffeldt (Hg.), S. 110-137.

Riegel, Mareike (2007): Sprachberatung im Kontext von Sprachpflege und im Verhältnis zu Nachschlagewerken. Unter besonderer Berücksichtigung der Sprachberatungsstelle des Wissen Media Verlags. Dissertation. Freiburg, S. 26-42. Internet: https://www.freidok. uni-freiburg.de/data/3593/ (Stand: 4.8.2016).

Schmidt, Jürgen Erich/Herrgen, Joachim (2011): Sprachdynamik: Eine Einführung in die moderne Regionalsprachenforschung. (= Grundlagen der Germanistik 49). Berlin.

Seelig, Babara (2002): Probleme und Tendenzen des deutschen Sprachgebrauchs. Ein Ergebnisbericht der Sprachberatungsstelle „Grammatisches Telefon Potsdam“ aus dem Zeitraum Juni 1997 bis Dezember 2000. In: Sprachreport 18, S. 2-7.

Szczepaniak, Renata (i.Vorb.): Die grammatikographische Praxis der Erfassung der reinen Genitivvariation. In: Fuß/Wöllstein (Hg.). 